\title{
Cadena de suministro aplicada en logística humanitaria: Caso batallón de movilidad de infantería de marina
}

\author{
Juan Pablo Lozano Borrero ${ }^{1}$
}

\section{Resumen}

Este artículo tiene como objetivo realizar un proceso de caracterización, evaluación, análisis y diagnóstico de las capacidades de respuesta del Batallón de Movilidad de Infantería de Marina $\mathrm{N}^{\circ}$ 1 ante la ocurrencia de una catástrofe de origen natural, así como la propuesta de un plan estratégico-logístico que busca mejorar estas capacidades para ser aplicadas bajo el concepto de la cadena de suministro en operaciones de ayuda humanitaria. Como resultados de esta investigación, se detectaron algunas debilidades existentes a nivel interno de la organización en cuanto a su capacidad de respuesta; de igual manera se halló que es aplicable para la Unidad el concepto de la cadena de suministro bajo un enfoque de Logística humanitaria que le permita explotar sus capacidades y desempeñar un papel más importante e influyente a nivel nacional e internacional.

\section{Palabras Claves}

Catástrofe natural, Plan Estratégico Logístico, Operaciones humanitarias.

\begin{abstract}
The objective of this article is to carry out a process of characterization, evaluation, analysis and diagnosis of the responsiveness of the Marine Infantry Mobility Battalion $\mathrm{N}^{\circ} 1$ in front of the occurrence of a natural catastrophe, as well as the proposal of a strategic-logistical plan that seeks to improve these capabilities to be applied under the concept of the supply chain in humanitarian aid operations. As a result of this research, some weaknesses were detected at the inside the organization in terms of its responsiveness; likewise, it was found that the concept of the supply chain is applicable to the Unit under a Humanitarian Logistics approach that allows it to exploit its capabilities and perform a more important and influential role at a national and international level.
\end{abstract}

\section{Keywords}

Natural catastrophe, Strategic Logistical Plan, Humanitarian operations.

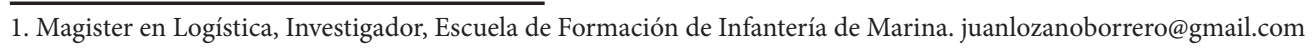




\section{Introducción}

Con el pasar de los años, y debido entre otros factores al cambio climático originado por la mano destructora del hombre, la humanidad se ve cada vez más expuesta y afectada por la ocurrencia de catástrofes y desastres de origen natural, que año tras año ocasionan no solo la pérdida de vidas humanas, sino que también generan destrucción y desolación en los lugares afectados. Los costos originados por este tipo de situaciones, además de afectar directamente la vida de las personas, también tienen gran incidencia en la economía de los países que lo sufren, puesto que al no tener establecidos los mecanismos y procedimientos para la prevención de los mismos se generan grandes pérdidas, y consecuentemente el proceso de reconstrucción se hace más lento, costoso y complejo.

Colombia, debido a su posición geográfica, la gran cantidad de fuentes hídricas que posee, y en general al variado relieve de su territorio, es un país que no se encuentra exento de la ocurrencia de este tipo de catástrofes, pues ya en años anteriores ha tenido sucesos trágicos e infortunados como los de Armero, Popayán, y el Eje Cafetero entre otros. Ante la incertidumbre y la imposibilidad de establecer con certeza las condiciones de forma, modo, hora y lugar en que ocurrirán este tipo de eventos, el país cuenta con la Unidad Nacional para la Gestión del Riesgo de Desastres la cual se encarga de dirigir, orientar y coordinar la Gestión del Riesgo de Desastres fortaleciendo las capacidades de las entidades públicas, privadas, comunitarias y de la sociedad, para contribuir al mejoramiento de la calidad de vida de las personas, a través del conocimiento del riesgo, su reducción y el manejo de los desastres. Así mismo a través del Ministerio de Defensa Nacional, a partir del año 2013 se estableció la Política para el Sector Defensa en Gestión del Riesgo de Desastres, la cual plasma unos objetivos, estrategias y lineamientos para la actuación de la Fuerza Pública ante la ocurrencia de este tipo de situaciones.

Para su caso particular, la Armada Nacional tiene proyectada, dentro del Comando de Infantería de Marina, la creación de una Unidad de Gestión del riesgo, la cual estará en cabeza

Ediciones EFIM de la coordinación e integración de todos los medios con los que se dispone no solo en temas de prevención de desastres, sino también para las actividades de respuesta y ayuda a los damnificados. Por su parte el Batallón de Movilidad de Infantería de Marina $\mathrm{N}^{\circ} 1$, como componente de la Armada, es una Unidad Táctica cuya misión es proveer movilidad táctica terrestre, despliegue de tropas y suministros, mediante el empleo de Patrullas Logísticas de Combate, con el propósito de contribuir al fortalecimiento de la capacidad de defensa, de la soberanía nacional y apoyar la participación en operaciones de paz y ayuda humanitaria, siendo la única unidad de la institución con este tipo de capacidades, por lo cual se hace necesario diagnosticar, evaluar y proponer algunas estrategias con el fin de mejorar su cadena de suministro y capacidad de respuesta ante la ocurrencia de una catástrofe de origen natural.

\section{Materiales y Método}

La investigación realizada es de tipo descriptivo y exploratorio, pues consiste en identificar el procedimiento utilizado por el Batallón de Movilidad de Infantería de Marina para responder en términos de logística y cadena de suministro ante una catástrofe natural, para posteriormente calcular su impacto y los beneficios desde la mejora en capacidad de respuesta, se realizó a partir de fuentes secundarias entre las que se encuentran documentos militares estratégicos, tesis de instituciones académicas, documentos gubernamentales y de $\mathrm{ONG}^{\prime}$ 's relacionadas con la atención de desastres y la gestión del riesgo, así como diferentes artículos y libros de bases de datos académicas. Así mismo, se utilizó la aplicación de encuestas y visitas de campo con el fin de tener una percepción más cercana a la realidad de la Unidad objeto de estudio.

Metodológicamente, este trabajo aborda los tipos de método Inductivo, puesto que inicia con un levantamiento de información acerca de la misión, organización y procedimientos de respuesta establecidos por el Batallón de Movilidad de Infantería de Marina $\mathrm{N}^{\circ} 1$ ante la ocurrencia de una catástrofe natural. Seguidamente, con base en dicha información y los resultados 
obtenidos en una encuesta aplicada a una población específica de esa Unidad, se establecen algunos factores determinantes de éxito para la organización, los cuales son evaluados interna y externamente a través de dos matrices, cuyos resultados nos presentan un diagnóstico de la situación actual en materia de capacidad de respuesta ante una situación de ese tipo. Finalmente, después de analizada esa información, se realiza la propuesta de un plan que incluye una serie de estrategias en pro del mejoramiento de las falencias detectadas, y que además propone la estructura de una cadena de suministro aplicada en logística humanitaria específicamente para esta Unidad de la Armada Nacional.

\section{Resultados y Discusión}

E1 Batallón de Movilidad de Infantería de Marina fue creado y activado el 22 de septiembre de 2011, mediante Disposición número 019 del Comando de la Armada Nacional de Colombia. Este documento en su artículo 13 precisa que:

"Modificase parcialmente el artículo $1^{\circ}$ de la Disposición $\mathrm{N}^{\circ} 021$ del 16 de diciembre de 2004 expedida por el comandante de la Armada Nacional, en el sentido de crear y activar en el Comando de Infantería de Marina (CIMAR), de la Brigada de Infantería de Marina $\mathrm{N}^{\circ} 1$ (BRIM1), el Batallón de Movilidad de Infantería de Marina $\mathrm{N}^{\circ} 1$ (BAMIM1)". (Armada Nacional, Disposición 019, 2011, p. 3).”

Así mismo más adelante en el artículo 14, establece su organización, puesto de mando y dependencia operacional, así:

"La organización del Batallón de Movilidad de Infantería de Marina $\mathrm{N}^{\circ} 1$ (BAMIM1) se sujetará a lo establecido en la Tabla de Organización y Equipo TOE No 3-0402- 0900, y tendrá su Puesto de Mando en Mahates (Bolívar). PARÁGRAFO.- Para efectos de mando y control operativo, el Batallón de Movilidad de Infantería de Marina $\mathrm{N}^{\circ} 1$ (BAMIM1), dependerá de la Brigada de Infantería de Marina N ${ }^{\circ} 1$ (BRIM1)". (Armada Nacional, Disposición 019,2011,p.4).”

Esta Unidad Táctica de la Infantería de Marina 34 de Colombia tiene como misión proveer movilidad táctica terrestre, despliegue de tropas y suministros, mediante el empleo de (PLC) Patrullas Logísticas de Combate (convoy), realizando actividades propias de mantenimiento y entrenamiento a los vehículos asignados; con el propósito de contribuir al fortalecimiento de la capacidad de defensa, de la soberanía nacional y apoyar la participación en operaciones de paz y ayuda humanitaria.

Para el cumplimiento de su misión y objetivos, el Batallón de Movilidad posee una estructura organizacional que se compone de un Comando de Batallón como cabeza de la organización, quien a su vez cuenta con una Plana Mayor Especial para su asesoramiento; de él se desprende un Segundo Comando, quien asume las funciones del primero durante su ausencia, y quien además se desempeña como Jefe de la Plana Mayor General, la cual está compuesta por seis secciones que cumplen funciones específicas en cuanto al manejo y administración de personal, inteligencia, operaciones, abastecimientos, acción integral y contrainteligencia. Finalmente, en la parte inferior de la estructura encontramos cuatro Compañías las cuales desempeñan tareas definidas: dos de transportes, una de mantenimiento y una de atención y prevención de desastres. Específicamente para el escenario de ayuda humanitaria, el Batallón de Movilidad además de la capacidad de transporte de su parque automotor, también cuenta con una Compañía especializada en atención y prevención de desastres. Esta Unidad Fundamental es una organización especializada de la Infantería de Marina en atención de desastres y emergencias alineada con la Dirección de Gestión del Riesgo de la Armada Nacional, con la capacidad de búsqueda y rescate marítimo y fluvial, BREC,APH,EDAN, enfocada en atender amenazas específicas de cambio climático, inundaciones y huracanes, así mismo participar integralmente en la Gestión del Riesgo, como parte de la política nacional en escenarios locales, regionales e internacionales. La estructura organizacional de esta Compañía se encuentra conformada por un Comandante de Compañía con su respectiva Maestría de Armas, un Segundo Comandante o Reemplazante de Compañía, un Pelotón de primera respuesta conformado por cuatro Anfibios ISSN: 2665-1513 
equipos: tres de Búsqueda y Rescate, y uno de Materiales Peligrosos, y un Pelotón de Estabilización conformado por un Equipo de Estabilización y un equipo de Parque Automotor.

Para dar respuesta a las diferentes contingencias o situaciones que se puedan presentar, el Batallón de Movilidad de Infantería de Marina $\mathrm{N}^{\circ} 1$ tiene establecidos unos procedimientos de respuesta para cada uno de sus componentes, en donde le asigna tareas, funciones y responsabilidades específicas encaminadas al correcto cumplimiento de la misión y a la mejora constante en el tiempo de respuesta.

Estos procedimientos se encuentran diseñados tanto para la planificación como para la ejecución por parte de las diferentes dependencias que componen la Unidad, así:

- Procedimiento de operaciones para la gestión del riesgo.

- Procedimiento de planificación para la gestión del riesgo.

- Procedimiento sección de operaciones para la gestión del riesgo.

- Procedimiento sección logística para la gestión del riesgo.

Todas las Instituciones poseen debilidades y fortalezas en sus distintas áreas funcionales, por lo que ninguna es igual de fuerte o débil en todas ellas. Al respecto, David (2003) señala que "Las fortalezas y las debilidades internas, junto con las oportunidades y las amenazas externas y una declaración de la misión definida, proporcionan una base para establecer objetivos y estrategias con la intención de aprovechar las fortalezas internas y superar las debilidades".

Para realizar el diagnóstico de la situación actual de la Cadena de Suministro aplicada en logística humanitaria del Batallón de Movilidad de Infantería de Marina $N^{\circ} 1$, se tuvo en cuenta la información investigada y levantada, así como los resultados de la encuesta realizada al personal de esa Unidad Militar. Así mismo, y con base en esa información, se aplicaron la Matriz de Evaluación de Factores Internos E.F.I., y la Matriz de Evaluación de Factores Externos E.F.E., las cuales permitieron realizar un análisis detaEdiciones EFIM llado de las fortalezas, debilidades, amenazas y oportunidades, de la capacidad de respuesta del Batallón ante una catástrofe de origen natural.

Tras el desarrollo de las matrices de evaluación tanto externo como interno (MEFE) (MEFI) encontramos una base académica “objetiva, práctica y viable" (Ponce Talancón, 2007, pág. 1) para abordar sistemáticamente las organizaciones y finalmente llegar a un diagnóstico ajustado a las realidades predominantes en la organización objeto de estudio.

La Matriz de Evaluación de Factores Internos del Batallón de Movilidad de Infantería de Marina, cuenta con 13 factores determinantes de éxito, 7 fortalezas y 6 debilidades. El valor obtenido de 2,471 indica un valor bastante cercano al promedio (2.5), lo que demuestra que la Unidad no es ni fuerte ni débil, sino que mantiene una respuesta media a las necesidades y requerimientos que tenga.

La Unidad cumple una misión que, aunque es un poco complejo por el tipo de servicio que presta, y por la incertidumbre de su "demanda" (ya que nunca se podrá saber con certeza la ocurrencia de una catástrofe), amerita acciones al interior de la misma que mejoren sustancialmente sus debilidades más apremiantes e influyentes, y que exploten mejor sus fortalezas para de esta manera convertirse en líder en ese sector dentro de las Fuerzas Militares de Colombia.

La Matriz de Evaluación de Factores Externos (MEFE) para el Batallón de Movilidad de Infantería de Marina $N^{\circ} 1$, cuenta con 10 factores determinantes de éxito, cuatro oportunidades y seis amenazas. El valor total de 2,711 indica una respuesta levemente por encima del promedio a las oportunidades y amenazas que el entorno presenta. En cuanto a las oportunidades, deben analizarse y aprovecharse de manera más efectiva, con el fin de aprovecharlas en lo máximo que se pueda, y lograr que la Unidad se integre e interactúe más con sus símiles a nivel nacional, y de esta manera ser más competitivos.

Con respecto a las amenazas, deben someterse a un riguroso análisis para poder diseñar las al- 
ternativas que le permitan tratar de establecer unos protocolos con los cuales esa incertidumbre con respecto a la ocurrencia y tipo de catástrofe pueda ser minimizada en aras de una respuesta más efectiva por parte del Batallón.

Con base en lo anterior, se puede establecer que si bien el batallón de Movilidad de Infantería de Marina $\mathrm{N}^{\circ} 1$, tiene establecida una organización adecuada, con una misión clara para la respuesta ante catástrofes naturales o provocadas a través de unos procedimientos ordenados y organizados para cada una de sus dependencias, presenta algunas falencias, dentro de ellas la no existencia de una cadena de suministro aplicada en logística humanitaria, que pueda facilitarle las acciones de almacenamiento, transporte y distribución de los elementos requeridos durante la respuesta a mencionadas emergencias.

Para la realización del Plan Estratégico-Logístico delBatallón de Movilidad de Infantería de Marina $\mathrm{N}^{\circ} 1$ se tuvieron en cuenta los siguientes aspectos: Condiciones Internas de la organización: en donde se hace énfasis en el contenido de la misión, la visión, y los objetivos de la organización.

Condiciones del entorno: Los distintos entornos están en su mayoría fuera del control de las organizaciones, por lo cual esas variables son de vital importancia y deben tenerse en cuenta en la formulación de estrategias.

Los recursos y la estrategia: Para la formulación del Plan Estratégico, es de vital importancia tener en cuenta el análisis externo (MEFE) basado en el entorno y el análisis interno (MEFI) basado las fortalezas sobre las que se espera obtener la ventaja. Así mismo concentrar la atención en la elaboración de una Matriz DOFA, que permita organizar y estructurar mejor los análisis y evaluaciones realizadas.

Formulación de estrategias: se deben diseñar las estrategias orientadas al logro de ventajas que permitan el mejoramiento de los planes y procesos de la organización.

Estrategia de diseño de la cadena de suministro: "Una vez analizado el direccionamiento estratégico de la compañía, se decide sobre las macro actividades cómo deben interactuar para lograr las utilidades esperadas por los socios. Una compañía debe asegurarse de que la configuración de la cadena de suministros apoye a los objetivos estratégicos e incrementa su superávit. Generalmente estas se toman a largo plazo de tal manera que con el tiempo generen valor agregado, y se logren los niveles de efectividad esperados". (Universidad Tecnológica de Pereira, Revista Scientia et Technica Año XVI, N 44, 2010, p. 94).

\section{Tabla 1. Estrategias para el logro de objetivos institucionales BAMIM 1}

\begin{tabular}{|c|c|}
\hline OBJETIVOS & ESTRATEGIAS \\
\hline \multirow{3}{*}{$\begin{array}{l}\text { Participar integralmente en } \\
\text { la Gestión del Riesgo, como } \\
\text { parte de la política nacional } \\
\text { en escenarios locales, regio- } \\
\text { nales e internacionales }\end{array}$} & $\begin{array}{l}\text { Promover, coordinar y } \\
\text { gestionar la participación } \\
\text { e integración de la Unidad } \\
\text { en el Sistema Nacional de } \\
\text { Gestión del Riesgo }\end{array}$ \\
\hline & $\begin{array}{l}\text { Promover y coordinar la } \\
\text { participación de la Unidad } \\
\text { en ejercicios y planes de } \\
\text { atención de desastres a nivel } \\
\text { internacional }\end{array}$ \\
\hline & $\begin{array}{l}\text { Nombrar personal de enlace } \\
\text { ante la oficina nacional de } \\
\text { gestión del riesgo }\end{array}$ \\
\hline \multirow{3}{*}{$\begin{array}{c}\text { Apoyar la atención de } \\
\text { emergencias a nivel nacional } \\
\text { oportuna y eficientemente }\end{array}$} & $\begin{array}{l}\text { Promover la participación de } \\
\text { la Unidad en situaciones rea- } \\
\text { les de atención de catástrofes }\end{array}$ \\
\hline & $\begin{array}{l}\text { Realizar y ejecutar planes de } \\
\text { reentrenamiento en atención } \\
\text { de desastres al personal de la } \\
\text { Unidad }\end{array}$ \\
\hline & $\begin{array}{l}\text { Realizar las coordinaciones } \\
\text { necesarias con los demás en- } \\
\text { tes encargados de la atención } \\
\text { de desastres a nivel local, } \\
\text { regional y nacional }\end{array}$ \\
\hline \multirow{4}{*}{ Fortalecer el talento humano } & Capacitación del personal \\
\hline & $\begin{array}{l}\text { Implementación de un siste- } \\
\text { ma de gestión por compe- } \\
\text { tencias }\end{array}$ \\
\hline & $\begin{array}{l}\text { Adecuar y fortalecer un ade- } \\
\text { cuado clima organizacional }\end{array}$ \\
\hline & $\begin{array}{l}\text { Desarrollar planes de } \\
\text { bienestar que incluyan a las } \\
\text { familias de los miembros de } \\
\text { la Unidad }\end{array}$ \\
\hline
\end{tabular}


Cadena de suministro aplicada en logística humanitaria: Caso batallón de movilidad de infantería de marina

\begin{tabular}{|c|c|}
\hline OBJETIVOS & ESTRATEGIAS \\
\hline \multirow{3}{*}{$\begin{array}{l}\text { Mejorar la capacidad estra- } \\
\text { tégica, técnica, tecnológica y } \\
\text { operativa de la Unidad para } \\
\text { ejercer de manera eficiente y } \\
\text { efectiva la respuesta ante una } \\
\text { catástrofe }\end{array}$} & $\begin{array}{l}\text { Gestionar la adquisición } \\
\text { de materiales y equipos de } \\
\text { última tecnología para la } \\
\text { atención de desastres }\end{array}$ \\
\hline & $\begin{array}{l}\text { Realizar y ejecutar planes de } \\
\text { comunicaciones estratégicas } \\
\text { con el fin de dar a conocer } \\
\text { las capacidades y apoyos } \\
\text { realizados por la Unidad }\end{array}$ \\
\hline & $\begin{array}{l}\text { Fortalecer el entrenamiento y } \\
\text { capacitación del personal }\end{array}$ \\
\hline \multirow{3}{*}{$\begin{array}{l}\text { Impulsar acciones para me- } \\
\text { jorar la eficiencia en la prepa- } \\
\text { ración para la respuesta y la } \\
\text { recuperación post desastre }\end{array}$} & $\begin{array}{l}\text { Efectuar continuamente } \\
\text { ejercicios y ensayos de los } \\
\text { planes de respuesta ante una } \\
\text { catástrofe }\end{array}$ \\
\hline & $\begin{array}{l}\text { Realizar mediciones conti- } \\
\text { nuas a los tiempos de res- } \\
\text { puesta de la Unidad ante la } \\
\text { ocurrencia de una catástrofe }\end{array}$ \\
\hline & $\begin{array}{l}\text { Realizar un diagnóstico } \\
\text { interno de la Unidad para } \\
\text { identificar las debilidades } \\
\text { que se puedan tener en los } \\
\text { procedimientos de respuesta }\end{array}$ \\
\hline \multirow{3}{*}{$\begin{array}{l}\text { Fomentar la investigación, } \\
\text { educación, comunicación y } \\
\text { gestión del conocimiento, } \\
\text { para una mayor conciencia } \\
\text { del riesgo en la Institución }\end{array}$} & $\begin{array}{l}\text { Realizar estudios tendientes } \\
\text { a la búsqueda de mejoras en } \\
\text { los procesos de respuesta de } \\
\text { la Unidad }\end{array}$ \\
\hline & $\begin{array}{l}\text { Fomentar la creación de } \\
\text { grupos de investigación para } \\
\text { la realización de proyectos de } \\
\text { mejora de la Unidad }\end{array}$ \\
\hline & $\begin{array}{l}\text { Realizar coordinaciones con } \\
\text { las escuelas de formación y } \\
\text { demás establecimientos de } \\
\text { carácter académico con el } \\
\text { fin de fomentar los estudios } \\
\text { sobre el tema }\end{array}$ \\
\hline
\end{tabular}

Fuente: elaboración propia

Arellano (2001), acerca de la Cadena Logística de los suministros en emergencia, precisa que "Los componentes de esta cadena logística, aunque no son necesariamente secuenciales y frecuentemente se desarrollan paralela y simultáneamente, deben ser considerados integralmente y no como actividades separadas, debido a su relación vinculante". Además, señala que su control no puede estar bajo responsabilidad de una sola persona, y que deberá componerse por las siguientes partes: el abastecimiento, el transporte., el almacenamiento, y la distribución.

\section{Figura 1. Propuesta cadena de suministro} BAMIM 1

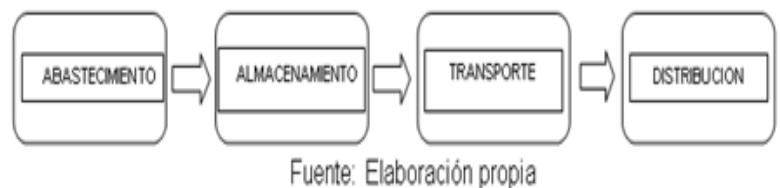

\section{Conclusiones}

El Batallón de Movilidad de Infantería de Marina $\mathrm{N}^{\circ} 1$ cuenta con una organización acorde a la Misión que le tiene asignada el Comando de la Armada Nacional. Así mismo tiene plenamente establecidos e identificados unos procedimientos de respuesta para cada uno de sus componentes, en donde les asigna tareas, funciones y responsabilidades específicas encaminadas al correcto cumplimiento de la misión y a la mejora constante en el tiempo de respuesta; estos procedimientos se encuentran diseñados tanto para la planificación como para la ejecución por parte de las diferentes dependencias y secciones que componen la Unidad, las cuales involucran a todos y cada uno de sus integrantes.

El Diagnóstico realizado a la Unidad utilizando las Matrices de Evaluación de Factores Internos y Externos nos permite concluir que la Unidad cumple una misión que, aunque es un poco compleja por el tipo de servicio que presta, y por la incertidumbre de su "demanda", amerita acciones al interior de la misma que mejoren sustancialmente sus debilidades más apremiantes e influyentes, y que exploten mejor sus fortalezas. Así mismo en cuanto a las oportunidades, estas deben ser aprovechadas al máximo para lograr que la Unidad se integre e interactúe más con sus símiles a nivel nacional, y de esta manera procurar ser más competitivos. Con respecto a las amenazas, estas deben someterse a un riguroso análisis para poder diseñar las alternativas que permitan tratar de establecer unos protocolos con los cuales esa incertidumbre con respecto a la ocurrencia y tipo de catástrofe pueda ser minimizada en aras de una respuesta más efectiva por parte del Batallón.

Por otra parte, con el diagnóstico también 
se puedo establecer que si bien el batallón de Movilidad de Infantería de Marina $\mathrm{N}^{\circ} 1$, tiene establecida una organización adecuada, para la respuesta ante catástrofes naturales o provocadas a través de unos procedimientos ordenados para cada una de sus dependencias, en ellos no se contempla el concepto explícito de una cadena de suministro aplicada en logística humanitaria, que pueda facilitarle las acciones de almacenamiento, transporte y distribución de los elementos requeridos durante la respuesta a mencionadas emergencias.

Finalmente se puede establecer que la propuesta del plan estratégico-logístico y el concepto de cadena de suministro con enfoque a la logística humanitaria que se plantea, es aplicable al Batallón de Movilidad de Infantería de Marina $\mathrm{N}^{\circ} 1$, puesto que se alinea a los objetivos estratégicos de la Armaba Nacional los cuales entre otras cosas pretenden impulsar este tipo de operaciones humanitarias no relacionadas con la guerra, las cuales empiezan a cobrar mucha importancia dentro del nuevo rol que la Institución debe desempeñar en un eventual escenario de posconflicto.

\section{Referencias bibliográficas}

Arellano, F. (2001). Logística y Gestión de suministros humanitarios en el sector salud. $\left(1^{\circ}\right.$ ed). Washington, Estados Unidos.

Armada Nacional. (2010). "Misión cumplida": Fuerzas Militares en Haití. Recuperado el: 20 de abril de 2015. Disponible en línea: https:// www.armada.mil.co/es/content/\%E2\%80\%9Cmisi $\% \mathrm{C} 3 \% \mathrm{~B} 3$-cumplida $\% \mathrm{E} 2 \% 80 \% 9 \mathrm{D}$-fuerzas - militares-en-hait \% C $3 \%$ AD .

Arroyoy Gaytán.(2011).Logísticaantedesastres, estrategia para actuar más rápido. Recuperado el: 28 de abril de 2015. Disponible en línea: http:// www.logisticamx.enfasis.com/notas/20693-.
Castillo, I. (2010). Logística Humanitaria: administración de la cadena de suministro. Recuperado el 29 de abril de 2015. Disponible en línea: http://besana111.com/ileana/cursos/2011/ IN4 $018 / \mathrm{pdf} /$ LogisticaHumanitaria.pdf

Centro de Información de las Naciones Unidas. (2015). Operaciones de paz de $\mathrm{Na}$ ciones Unidas. Recuperado el 30 de abril de 2015. Disponible en línea: http://www. cinu.org.mx/temas/paz_seguridad/pk.htm

Chopra y Meindl. (2008). Administración de la cadena de suministro. Estrategia, planeación y operación. ( $3^{\circ}$ ed). Naucalpan de Juárez, México: Pearson educación.

Conoir, Y. (2002). La conducción de operaciones de ayuda humanitaria: Principios para la intervención y administración. Recuperado el: 20 de abril de 2015. Disponible en línea: http://cdn.peaceopstraining. org/course_promos/humanitarian_relief ops/humanitarian_relief_ops_spanish.pdf.

Corporación AVRE. (2004). La atención humanitaria en el contexto colombiano. Recuperado el: 29 de abril de 2015. Disponible en línea: http://www.corporacionavre. org/files/pdf/LibroNaranja/naranja_2.pdf.

David, F. (2003). Conceptos de Administración Estratégica. La evaluación Interna. ( $9^{\circ}$ ed). Naucalpan de Juárez, México: Pearson educación.

Difusión y aplicación tareas y roles Fuerzas Militares. (Directiva permanente $\left.\quad \mathrm{N}^{\circ} 003\right)$. (2009, 5 enero).

Gestiopolis. (2004). La cadena de suministro en la gestión logística. Recuperado el: 26 de abril de 2015. Disponible en línea: http://www.gestiopolis.com/la-cadena-de-suministro-en-la-gestion-logistica/.

Hernández y Ruiz. (2012). Desarrollo de un Plan Estratégico de Logística para la Empresa Aconquistar S.A.S. Trabajo de grado no publicado, Universidad del Rosario Bogotá, Colombia. 
Ministerio de Defensa Nacional. (2009). La Fuerza Pública y los retos del futuro. $\left(3^{\circ}\right.$ ed). Bogotá, Colombia: Imprenta Nacional

Ministerio de Defensa Nacional. (2011). Política Integraldeseguridadydefensa paralaprosperidad. ( $1^{\circ}$ ed). Bogotá, Colombia: Imprenta Nacional.

Ministerio de Defensa Nacional. (2013). Política para el sector Defensa en Gestión del Riesgo de Desastres. ( $1^{\circ}$ ed). Bogotá, Colombia: Imprenta Nacional.

Nuñez, Rey \& Thieux. (2007). Fuerzas Armadas y acción humanitaria: debates y propuestas. Recuperado el: 22 de abril de 2015. Disponible en línea: http://www.iecah.org/web/images/stories/publicaciones/libros/descargas/DT13.pdf.

Proyecto Esfera. (2011). Carta Humanitaria y normas mínimas para la respuesta humanitaria. $\left(3^{\circ} \mathrm{ed}\right)$. Bourton on Dunsmore, Reino Unido: Practical Action Publishing.

Rojas y Solano. (2012). Propuesta de protocolo para la creación de un Batallón de Infantería de Marina para el desarrollo de operaciones humanitarias y sostenimiento de infraestructura en la Armada Nacional. Trabajo de grado posgrado no publicado, Escuela de Ingenieros Militares Bogotá, Colombia.

Universidad Tecnológica de Pereira. (2010). Planeación Estratégica Logística para un Holding Empresarial. Revista Scientia et Technica Año XVI, N ${ }^{\circ} 44$. 